CoRollame I. Si une fonction $f(z)$ de la famille $C$ s'annule pour une suite de points $a_{1}, a_{2}, \ldots$ telle que

$$
\sum_{j=1}^{\infty}\left\{1-\left|a_{j}\right|\right\}^{2}=+\infty
$$

elle est identiquement nulle.

CorolraIRE II. Si une suite de fonctions pour lesquelles on a l'inégalité (10) converge en une infinité de points ayant au moins un point limite intérieur au cercle unité, la suite converge presque uniformément dans l'intérieur de ce cercle.

\section{Propriétés des points extrémaux des ensembles plans et leur application à la représentation conforme}

\author{
par F. LeJA (Krakow)
}

1. Introduction. Soit $E$ un ensemble fermé et borné de points du plan, $D$ un domaine quelconque contenant $E, p(z)$ une fonction holomorphe dans $D$ ne s'annulant pas dans ce domaine ${ }^{1}$ ) et $f(z)$ une fonction réelle, définie et continue dans $E$.

Désignons par $\omega(z, \zeta)$ l'expression

$$
\omega(z, \zeta)=\frac{|z-\zeta|}{|p(z) p(\zeta)| \exp [f(z)+f(\zeta)]},
$$

par $\zeta^{(n)}$ un système de $n+1$ points différents quelconques $\zeta_{0}, \zeta_{1}, \ldots, \zeta_{n}$ de $E$, par $V\left(\zeta^{(n)}, \omega\right)$ et $\Delta_{j}\left(\zeta^{(n)}, \omega\right)$ les produits

$$
\begin{aligned}
& V\left(\zeta^{(n)}, \omega\right)=\prod_{n \leqslant j<k \leqslant n} \omega\left(\zeta_{j}, \zeta_{k}\right), \\
& \Delta_{j}\left(\xi^{(n)}, \omega\right)=\prod_{\substack{k=0 \\
k \neq j}} \omega\left(\zeta_{j}, \zeta_{k}\right), \quad j=0,1, \ldots, n
\end{aligned}
$$

et soit $V_{n}(E, \omega)$ la borne supérieure de $V\left(\zeta^{(n)}, \omega\right)$, lorsque les points du système $\zeta^{(n)}$ varient arbitrairement dans $E$. D'autre part, soit

$$
x^{(n)}=\left\{x_{0}^{(n)}, x_{1}^{(n)}, \ldots, x_{n}^{(n)}\right\}
$$

un système de points de $E$ pour lequel

$$
\nabla\left(x^{(n)}, \omega\right)=V_{n}(E, \omega)=\sup _{\zeta^{(n)} \in E} \nabla\left(\zeta^{(n)}, \omega\right) .
$$

Les indices inférieurs des points $x_{j}^{(n)}$ peuvent toujours être choisis de manière qu'on ait

$$
\Delta_{0}\left(x^{(n)}, \omega\right) \leqslant \Delta_{1}\left(x^{(n)}, \omega\right) \leqslant \ldots \leqslant \Delta_{n}\left(x^{(n)}, \omega\right) .
$$

Les points $d u$ sytème (3) remplissant les conditions (4) et (5) sont dits points extrémaux de rang $n$ de $E$ par rapport à la fonction $\omega(z, \zeta)$, dite fonction génératrice ou distance généralisée des points $z$ et $\zeta$.

1) La fonction $p(z)$ peut être multiforme lorsque $D$ est multiplement connexe, mais nous supposerons que son module $|p(z)|$ est uniforme. 
Le produit $\nabla\left(x^{(n)}, \omega\right)$ a $n(n+1) / 2$ facteurs. On sait [3] que la moyenne géométrique

$$
\left[\nabla\left(x^{(n)}, \omega\right)\right]^{2 / n(n+1)}, \quad n=1,2, \ldots
$$

converge vers une limite non négative

$$
\lim _{n \rightarrow \infty}\left[\nabla\left(x^{(n)}, \omega\right)\right]^{2 / n(n+1)}=v(E,(i))
$$

dite écart de l'ensemble $E$ par rapport à la fonction $\omega(z, \zeta)$. Pareillement la moyenne $\Delta_{0}\left(x^{(n)}, \omega\right)^{1 / n}, n=1,2, \ldots$ converge vers la même limite ${ }^{2}$ )

$$
\left.\lim _{n \rightarrow \infty} \sqrt[n]{\Delta_{0}\left(x^{(n)}\right.}, \omega\right)=v(E, \omega) .
$$

Dans le cas où $\omega(z, \zeta)$ se réduit à la distance $|z-\zeta|$, c'est-à-dire lorsque $p(z) \equiv 1$ et $f(z) \equiv 0$, l'écart $v(E,|z-\zeta|)$ est appelé aussi diamètre transfini ou capacité de l'ensemble $E$. Nous supposerons dans la suite que $v(E,|z-\zeta|)>0$.

Désignons par $L^{(j)}\left(z, \zeta^{(n)}\right), j=0,1, \ldots, n$, les polynômes de Lagrange

$$
L^{(j)}\left(z, \zeta^{(n)}\right)=\prod_{\substack{k=0 \\ k \neq j}}^{n}\left(z-\zeta_{k}\right) /\left(\zeta_{j}-\zeta_{k}\right)
$$

liés au système $\zeta^{(n)}$, par $\Phi^{(j)}\left(z, \zeta^{(n)}\right), j=0,1, \ldots, n$, les fonctions

(7) $\quad \Phi^{(j)}\left(z, \zeta^{(n)}\right)=L^{(j)}\left(z, \zeta^{(n)}\right)\left[p\left(\zeta_{j}\right) / p(z)\right]^{n} \exp \left[n f\left(\zeta_{j}\right)\right], \quad j=0,1, \ldots, n$,

et par

$$
\Phi_{n}(z, E, \omega)=\inf _{\zeta^{\left(n^{\prime} \in E\right.} \in}\left\{\max _{(j)}\left|\Phi^{(j)}\left(z, \zeta^{(n)}\right)\right|\right\}, \quad n=1,2, \ldots
$$

la borne inférieure du plus grand des modules $\left|\Phi^{(j)}\left(z, \zeta^{-(n)}\right)\right|, j=0,1, \ldots, n$ lorsque, $z \in D$ et $n$ étant fixes, le système $\zeta^{(n)}$ varie arbitrairement dans $E$. D'autre part, formons les fonctions

$$
\Phi^{(j)}\left(z, x^{(n)}\right), \quad j=0,1, \ldots, n,
$$

correspondant au système extrémal $(3)$ et désignons par $E^{*}$ l'ensemble des points d'accumulation de la suite triangulaire

$$
\begin{aligned}
& x_{0}^{(1)}, x_{1}^{(1)}, \\
& x_{0}^{(2)} ; x_{1}^{(2)}, x_{2}^{(2)}, \\
& x_{0}^{(3)}, x_{1}^{(3)}, x_{2}^{(3)}, x_{3}^{(3)} \\
& \ldots \ldots \ldots
\end{aligned}
$$

2) La démonstration de ce fait dans le cas général (où la fonction $\omega$ est quelconque) n'est pas publiée, mais on sait que $\varlimsup_{n \rightarrow \infty} \sqrt[n]{\Delta_{0}\left(x^{(n)}, \omega\right)}=v(E, \omega)$.
Il est clair que l'ensemble $E^{*}$ est fermé et contenu dans $E$ et que parmi les fonctions (9) la condition (5) fait distinguer $\Phi^{(0)}\left(z, x^{(n)}\right)$ $[4])$ :

Nous aurons à nous appuyer sur les résultats suivants (ef. [1] et

Si $v(E,|z-\zeta|)>0$, la suite $\left\{\sqrt[n]{\Phi_{n}(z, E, \omega)}\right\}$ converge en tout point du domaine $D$

$$
\lim _{n \rightarrow \infty} \sqrt[n]{\Phi_{n}(z, E, \omega)}=\Phi(z, E, \omega), \quad z \in D .
$$

Pareillement la suite $\left\{\sqrt[n]{\left|\Phi^{0}\left(z, x^{(n)}\right)\right|}\right\}$ converge dans $D$ en dehors de l'ensemble $E^{*}$ vers la même limite

$$
\left.\lim _{n \rightarrow \infty} \sqrt[n]{\mid \Phi^{(0)}\left(z, x^{(n)}\right.}\right) \mid=\Phi(z, E, \omega), \quad z \in D-E^{*},
$$

la convergence (11) étant uniforme dans le voisinage de tout point $z \epsilon D-\boldsymbol{E}^{*}$.

La limite $\Phi(z, E, \omega)$ sera dite fonction extrémale du domaine $D_{4}^{*}$ par rapport à la fonction génératrice $\omega(z, \zeta)$. Le but de ce travail est d'examiner certaines propriétés de l'écart $v(E, \omega)$, de l'ensemble $E^{*}$ et de la fonction extrémale $\Phi(z, E, \omega)$ et d'appliquer cette étude au problème de la représentation conforme des domaines plans.

Remarque. Soient $m$ et $M$ les bornes inférieure et supérieure de $f(z)$ et $l$ et $L$ celles de $|p(z)|$ lorsque $z$ varie dans $E$. Alors $l>0$ et

$$
\frac{|z-\zeta|}{L^{2} e^{2 M}} \leqslant \omega(z, \zeta) \leqslant \frac{|z-\zeta|}{l^{2} \cdot e^{2 m}}
$$

ce qui entraîne l'inégalité

$$
\frac{v(E,|z-\zeta|)}{L^{2} e^{2 M}} \leqslant v(E, \omega) \leqslant \frac{v(E,|z-\zeta|)}{l^{2} e^{2 m}} .
$$

Par suite l'écart $v(E, \omega)$ est positif, quels que soient $p(z)$ et $f(z)$, lorsque $v(E,|z-\zeta|)$ est positif.

Remarquons encore que si $p(z) \equiv 1$ et $f(z) \equiv \lambda$, où $\lambda$ est une constante réelle quelconque, alors

$$
v(E, \omega)=v(E,|z-\zeta|) / e^{2 \lambda}
$$

2. Quelques propriétés de l'écart et de la fonction extrémale. L'écart $v(E, \omega)$ dépend des fonctions $p(z)$ et $f(z)$ et sera désigné aussi par $v(E, p, f)$. Lorsque $E$ varie en restant fermé et borné et les fonctions $p$ et $f$ restent fixes, il résnlte immédiatement de (4) et (6) que

$$
v\left(E^{\prime}, p, f\right) \leqslant v(E, p, f) \quad \text { si } \quad E^{\prime} \subset E .
$$

Faisons maintenant varier $f$ sans changer $E$ et $p$. Je dis que: 
I. La fonctionnelle $v(E, p, f)$ varie continuement aveo $f$; elle décroît (au sens large) lorsque $f$ crôt ${ }^{3}$ ).

Démonstration. Il s'agit prouver qu'à chaque $\varepsilon>0$ correspond un nombre $\delta>0$ tel que, si deux fonctions $f(z)$ et $f(z)$ satisfont dans $E$ à l'inégalité $|\bar{f}-f|<\delta$, on ait

$$
|v(E, p, \bar{f})-v(E, p, f)|<\varepsilon .
$$

Soient $x^{(n)}=\left\{x_{0}, x_{1}, \ldots, x_{n}\right\}$ et $\bar{x}^{(n)}=\left\{\bar{x}_{0}, \bar{x}_{1}, \ldots, \bar{x}_{n}\right\}$ denx systèmes de points extrémaux de rang $n$ de $E$ respectivement par rapport à $\omega(z, \zeta)$ et $\bar{\omega}(z, \zeta)$, où

$$
\bar{\omega}(z, \zeta)=\frac{|z-\zeta|}{|p(z) p(\zeta)| \exp [\bar{f}(z)+\bar{f}(\zeta)]} .
$$

Posons

$$
\Delta f=\sum_{k=0}^{n}\left[\bar{f}\left(x_{k}\right)-f\left(x_{k}\right)\right]
$$

Si $|\bar{f}(z)-f(z)|<\delta$ dans $E$ où $\delta>0$ est quelconque, on a $|\Delta f| \leqslant(n+1) \delta$ Mais, d'après (4), on a

$$
V\left(\bar{x}^{(n)}, \bar{\omega}\right) \geqslant V\left(x^{(n)}, \bar{\omega}\right)=V\left(x^{(n)}, \omega\right) e^{-n \Delta t}
$$

done l'inégalité $|\bar{f}(z)-f(z)|<\delta$ entraîne la suivante

$$
V\left(\bar{x}^{(n)}, \bar{\omega}\right)>V\left(x^{(n)}, \omega\right) e^{-n(n+1) \delta}
$$

d'où l'on déduit: $v(E, p, \bar{f})>v(E, p, f) e^{-2 \delta}$. Pareillement, si $|\bar{f}-f|<\delta$ sur $E$ on a $v(E, p, f)>v(E, p, \bar{f}) e^{-2 \delta}$ et par suite les quantités $v=$ $=v(E, p, f)$ et $\bar{v}=v(E, p, \bar{f})$ satisfont aux inégalités

$$
v\left(e^{-2 \delta}-1\right)<\bar{v}-v<v\left(e^{2 \delta}-1\right)
$$

d'où l'on conclut que l'inégalité (12) est satisfaite lorsque $|\bar{f}-f|<\delta$ et $\delta$ est suffisamment petit.

Pour terminer la démonstration considérons l'indentité

$$
V\left(\zeta^{(n)}, \bar{\omega}\right)=V\left(\zeta^{(n)}, \omega\right) e^{-n \Delta t}, \quad \text { où } \quad \Delta f=\sum_{k=0}^{n}\left[\bar{f}\left(\zeta_{k}\right)-f\left(\zeta_{k}\right)\right] .
$$

Si $\bar{f} \geqslant f$ en tout point de $E$, on a $\Delta f \geqslant 0$ et par suite $V\left(\zeta^{(n)}, \bar{\omega}\right) \leqslant V\left(\zeta^{(n)}, \omega\right)$, ce qui entraîne l'inégalité $v(E, \bar{\omega}) \leqslant v(E, \omega)$.

s) C'est-à-dire lorsque $f(z)$ ne diminue en aucun point de $t$.

La fonction extrémale $\Phi(z, E, \omega)$ sera aussi désignée par $\Phi(z, E, p, f)$ Elle jouit des propriétés suivantes:

$$
\begin{aligned}
& \left.\Phi\left(z, E^{\prime}, p, f\right) \geqslant \Phi(z, E, p, f) \quad \text { si } \quad E^{\prime} \subset E^{4}\right), \\
& \Phi(z, E, p, f) \leqslant e^{f(z)} \quad \text { pour } \quad z \in E, \\
& \Phi(z, E, p, f) \geqslant l e^{m} /|p(z)| \quad \text { pour } \quad z \in D,
\end{aligned}
$$

où l'on a posé $l=\min _{z_{\theta} E}|p(z)|, m=\min _{z_{\theta} E} f(z)$.

En effet, l'inégalité (13) résulte immédiatement de (8) et (10). Pour prouver (14) remplaçons dans le système extrémal (3) le point $x_{j}^{(n)}$ par un point quelconque $z \epsilon E$ et désignons le système obtenu par $\bar{x}^{(n)}$. D'après (4) on a

$$
V\left(\bar{x}^{(n)}, \omega\right) \leqslant V\left(x^{(n)}, \omega\right)
$$

et cette inégalité entraîne immédiatement la suivante

$$
\left|\Phi^{(j)}\left(z, x^{(n)}\right)\right| \leqslant e^{n t(z)}, \quad j=0,1, \ldots, n,
$$

et comme, d'après (8),

$$
\Phi_{n}(z, E, \omega) \leqslant \sum_{j=0}^{n}\left|\Phi^{(j)}\left(z, x^{(n)}\right)\right|
$$

on voit que $\Phi_{n}(z, E, \omega) \leqslant(n+1) e^{n f(z)}, z \in E$, ce qui entraîne l'inégalité (14).

Soit $\xi^{(n)}=\left\{\xi_{0}, \xi_{1}, \ldots, \xi_{n}\right\}$ un système de points de $E$ pour lequel $\Phi_{n}(z, E, \omega)=\max \left|\Phi^{(j)}\left(z, \xi^{(n)}\right)\right|$. Il résulte de la formule (7) que

$$
\max _{(j)}\left|\Phi^{(j)}\left(z, \xi^{(n)}\right)\right| \geqslant\left(\max _{(j)}\left|L^{(j)}\left(z, \xi^{(n)}\right)\right|\right) \frac{\left(l e^{m}\right)^{n}}{|p(z)|^{n}}
$$

et comme $\sum_{j=0}^{n} L^{(j)}\left(z, \xi^{(n)}\right)=1$ on a, quel que soit $z$,

et par suite

$$
\max _{(f)}\left|L^{(j)}\left(z, \xi^{(n)}\right)\right| \geqslant 1 /(n+1)
$$

$$
\sqrt[n]{\Phi_{n}(z, E, \omega)} \geqslant \frac{1}{\sqrt[n]{n+1}} \cdot \frac{l e^{m}}{|p(z)|}
$$

ce qui entraîne l'inégalité (15).

Soit $z_{0}$ un point de l'ensemble $E$. Nous dirons que $E$ jouit en $z_{0}$ de la propriété $W$, si à chaque $\varepsilon>0$ correspond un nombre $\delta>0$ tel que, pour toute suite de polynômes $\left\{P_{n}(z)\right\}$ de degré $\leqslant n$, uniformément bornés dans $E$

$$
\varlimsup_{n \rightarrow \infty} \sqrt[n]{\left|P_{n}(z)\right|}<1+\varepsilon
$$

4) On suppose que $E^{\prime}$ est fermé, comme $E$, et que le diamètre transfini $v$ ( $E^{\prime}$, $|z-\zeta|)$ est positif, ce qui assure l'existence de la fonction extrémale $\Phi\left(z, E^{\prime}, \omega\right)$. 
dans le voisinage $\left|z-z_{0}\right|<\delta$. On sait que $E$ jouit de la propriété $W$ en tout point d'un continu quelconque $C \subset E$ et n'en jouit pas en des points isolés de celui-ci.

D'après (15) la fonction $\Phi(z, E, p, f)$ est positive en tout point du domaine $D$ et, les fonctions $\Phi^{(0)}\left(z, x^{(n)}\right), n=1,2, \ldots$ étant analytiques dans $D$ et la convergence (11) étant uniforme, il résulte de (11) que la fonction $\log \Phi(z, E, p, f)$ est harmonique dans $D-E^{*}$. Sur $E^{*}$ elle jouit des propriétés suivantes:

II. La fonction $\Phi(z, E, p, f)$ reste bornée dans le voisinage de tout point $z_{0} \in E^{*}$, admet en $z_{0}$ la valeur

$$
\Phi\left(z_{0}, E, p, f\right)=e^{f\left(z_{0}\right)}
$$

et, si l'ensemble $E$ jouit en $z_{0}$ de la propiriété $W, \Phi(z, E, p, f)$ reste continue en ce point.

La démonstration de ce théorème est analogue à celle du théorème IV que j'ai donnée dans mon travail [4].

Désignons par $D_{\infty}(E)$ le plus grand domaine non borné contenu dans l'ensemble complémentaire de $E$ et supposons que les fonctions $p(z)$ et $f(z)$ soient constantes $p(z) \equiv 1, f(z) \equiv 0$. Alors le domaine $D$ est le plan entier, la fonction $\log \Phi(z, E, 1,0)$ est harmonique partout en dehors de $E^{*}$ et s'annule sur $E^{*}$. Comme

$$
\Phi^{(0)}\left(z, x^{(n)}\right)=\prod_{k=1}^{n} \frac{z-x_{k}^{(n)}}{x_{0}^{(n)}-x_{k}^{(n)}}
$$

la différence $\log \Phi(z, E, 1,0)-\log |z|$ tend d'après (11) vers une limite finie lorsque $|z| \rightarrow \infty$. D'autre part, on déduit du principe de maximum que les points extrémaux sont situés sur la frontière $F$ du domaine $D_{\infty}(E)$ et que $E^{*}$ couvre $F$ tout entier $\left.{ }^{5}\right)$. Par conséquent [2] $\log \Phi(z, E, 1,0)$ est la fonction de Green du domaine $D_{\infty}(E)$ de pôle à l'infini. En dehors de ce domaine la fonction $\log \Phi(z, E, 1,0)$ est identiquement nulle.

3. Fonctions analytiques liées à la fonction extrémale $\Phi(z, E, \omega)$. Soit $I I$ le plan entier. La différence $\Pi-E^{*}$ est une somme de domaines disjoints dont un et un seul est non borné. Désignons ce dernier domaine par $D_{\infty}=D_{\infty}\left(E^{*}\right)$ et les autres (s'ils existent) par $D_{1}, D_{2}, \ldots$; on aura

$$
\Pi-E^{*}=D_{\infty}+D_{1}+D_{2}+\ldots
$$

$\left.{ }^{5}\right)$ Si un point $z_{0}$ de $F^{*}$ n'appartenait pas à $E^{*}$, la fonction $\log \Phi(z, E, 1,0)$ serait harmonique en $z_{0}$ et $y$ atteindrait son minimum $0-$ ce qui est impossible.
Pareillement la différence $D-E^{*}$ est une somme de domaines dont un et un seul est contenu dans chaque $D_{k}$. Désignons le domaine partiel de $D-E^{*}$ contenu dans $D_{l k}$ par $\Lambda_{k}$; on aura

$$
D-E^{*}=\Delta_{\infty}+\Delta_{1}+\Delta_{2}+\ldots
$$

Choisissons dans chaque domaine $\Delta_{k}$ un point fixe quelconque $a_{k}$ et désignons par $\varphi_{n}\left(z, \Delta_{k}\right), k=\infty, 1,2, \ldots$, la fonction analytique définie dans $\Delta_{k}$ par la formule

$$
\varphi_{n}\left(z, \Delta_{k}\right)=e^{i \Theta_{n}} \sqrt[n]{\Phi^{(0)}\left(z, x^{(n)}\right)}, \quad n=1,2, \ldots
$$

où la branche du radical et le nombre réel $\Theta_{n}$ sont choisis de manière que la valeur de $\varphi_{n}\left(z, \Delta_{l k}\right)$ au point $z=a_{k}$ soit positive $\varphi_{n}\left(a_{k}, \Delta_{k}\right)>0$.

Il résulte de (1.1) que la suite (17) converge au point $z=a_{k}$ et que la suite des modules $\left|\varphi_{n}\left(z, \Delta_{k}\right)\right|$ converge dans le domaine $\Delta_{k}$ vers $\Phi(z, E, \omega)$, d'où l'on. conclut que:

III. La suite (17), où $k=\infty, 1,2, \ldots$ est fixé, converge dans le domaine $\Delta_{k}$ vers une fonction

$$
\lim _{n \rightarrow \infty} \uparrow_{n}\left(z, \Delta_{k}\right)=\varphi\left(z, \Delta_{k}\right)
$$

analytique dans $\Delta_{k}$ de module

$$
\left|\varphi\left(z, \Delta_{k}\right)\right|=\Phi(z, E, \omega), \quad z \epsilon \Delta_{k},
$$

la convergence étant uniforme dans le voisinage de tout point $z \in \Delta_{k}$.

La fonction $\varphi\left(z, \Delta_{k}\right)$ sera aussi désignée, plus précisément, par

$$
\varphi\left(z, \Delta_{k}, p, f\right), \quad k=\infty, 1,2, \ldots
$$

Il est clair que cette fonction peut être uniforme ou multiforme dans le domaine $\Delta_{k}$, mais son module est toujours uniforme.

La frontière de l'ensemble ouvert (16) est la somme de la frontière $F_{1}$ de l'ensemble fermé $E^{*}$ et de la frontière $F_{2}$ du domaine $D$. L'allure des fonctions $\varphi\left(z, \Delta_{k}\right), k=\infty, 1,2, \ldots$ dans le voisinage de $F_{1}$ est caractérisée par le théorème II et, dans le voisinage de $F_{2}$, par le suivant:

IV. Le produit $\varphi\left(z, \Delta_{k}\right) p(z)$, ò̀ $k=\infty, 1,2, \ldots$, est prolongeable analytiquement au domaine $D_{k}$ et reste borné dans $D_{k}$ si $k \neq \infty$, tandis que le produit $\varphi\left(z, \Delta_{\infty}\right) p(z)$ prolongé à $D_{\infty}$ n'est pas borné dans le voisinage du seul point $z=\infty$ et admet dans ce voisinage un développement de la forme

$$
p\left(z, \Delta_{\infty}\right) p(z)=\gamma_{-1} z+\gamma_{0}+\gamma_{1} z^{-1}+\gamma_{2} z^{-2}+\ldots,
$$

où le coefficient $\gamma_{-1}$ est différent de zéro.

Démonstration. Pour simplifier l'écriture désignons le système extrémal (3) $x^{(n)}=\left\{x_{0}, x_{1}, \ldots, x_{n}\right\}$ et posons

$$
w\left(x^{(n)}\right)=\left|\left(x_{0}-x_{1}\right)\left(x_{0}-x_{2}\right) \ldots\left(x_{0}-x_{n}\right)\right|^{1 / n}\left|p\left(x_{0}\right) e^{f\left(x_{0}\right)}\right|^{-1} .
$$


D'après la formule (7) on a

$$
\Phi^{(0)}\left(x^{(n)}, \omega\right)=\left(\prod_{k=1}^{n} \frac{z-x_{k}}{x_{0}-x_{k}}\right)\left[\frac{p\left(x_{0}\right) e^{f\left(x_{0}\right)}}{p(z)}\right]^{n},
$$

donc, d'après (17),

(21) $\quad \varphi_{n}\left(z, \Delta_{k}\right) p(z)=\frac{e^{i \theta}}{w\left(x^{(n)}\right)} \sqrt[n]{\left(z-x_{1}\right)\left(z-x_{2}\right) \ldots\left(z-x_{n}\right)}, \quad n=1,2, \ldots$

où $\Theta_{n}^{\prime}$ est un nombre réel convenablement choisi ot $k=\infty, 1,2, \ldots$

Les membres gauches des identités (21) convergent dans le domaine $\Delta_{k}$ vers $\varphi\left(z, \Delta_{k}\right) p(z)$ et les membres droits sont uniformément bornés dans chaque domaine borné, car les termes de la suite $\left\{w\left(x^{(n)}\right)\right\}$ sont plus grands qu'un nombre positif $\left.{ }^{6}\right)$. Il s'ensuit que la fonction $p\left(z, \Delta_{k}\right) p(z)$ se prolonge analytiquement au domaine $D_{k} \supset \Delta_{k}$ car, quel que soit $n$, le membre droit de (21) est une fonction analytique dans $D_{l}$. La fonction prolongée $\varphi\left(z, \Delta_{k}\right) p(z)$ reste bornée dans $D_{k}$ si $k=1,2, \ldots$, car les domaines $D_{1}, D_{2}, \ldots$ sont bornés.

Soit $R$ un nombre positif assez grand pour que le cercle $|z| \leqslant R$ contienne l'ensemble $E$. Dans le domaine $\Delta_{\infty}$ on a identiquement

$$
\frac{\varphi_{n}\left(z, \Delta_{\infty}\right) p(z)}{z}=\frac{e^{i \theta_{n}^{\prime}}}{w\left(x^{(n)}\right)} \sqrt[n]{\left(1-\frac{x_{1}}{z}\right)\left(1-\frac{x_{2}}{z}\right) \ldots\left(1-\frac{x_{n}}{z}\right)}
$$

$$
n=1,2, \ldots
$$

Le second membre de cette identité est analytique et uniformément borné dans le domaine $D_{\infty}$ et, comme il converge dans $\Delta_{\infty} \subset D_{\infty}$, la convergence a lieu dans le domaine $D_{\infty}$, ce qui prouve que la fonction $\varphi\left(z, \Delta_{\infty}\right) p(z)$ se prolonge au domaine $D_{\infty}$. D'autre part, quel que soit $n$, le second membre de (22) est développable dans le domaine $|z|>R$ en une série de la forme

$$
\gamma_{-1}^{(n)}+\frac{\gamma_{0}^{(n)}}{z}+\frac{\gamma_{1}^{(n)}}{z^{2}}+\ldots, \quad \text { où } \quad\left|\gamma_{-1}^{(n)}\right|=\frac{1}{w\left(x^{(n)}\right)}
$$

et comme la suite de ces séries converge uniformément dans le domaine $|z|>R$ vers $\varphi\left(z, \Delta_{\infty}\right) p(z) / z$, les suites des coefficients $\left\{\gamma_{k}^{(n)}\right\}$ convergent
vers des limites déterminées

$$
\lim _{n \rightarrow \infty} \gamma_{k}^{(n)}=\gamma_{k}, \quad k=-1,0,1, \ldots
$$

0) $\operatorname{Car} w\left(x^{(n)}\right)=\sqrt[n]{\Delta_{0}\left(x^{(n)}, \omega\right)}\left[\prod_{k=1}^{n}\left|p\left(x_{k}\right) e^{f\left(x_{k}\right)}\right|\right]^{1 / n} \geqslant l e^{m} \sqrt[n]{\Delta_{0}\left(x^{(n)}, \omega\right)}$ et $\sqrt[n]{\Delta_{0}\left(x^{(n)}, \omega\right)}$ $\rightarrow v(E, \omega)>0$. et la fonction prolongée $\varphi\left(\hat{z}, \Delta_{\infty}\right) p(z)$ se développe en la série (18), où

$$
\left|\gamma_{-1}\right|=\lim _{n \rightarrow \infty}\left[1 / w\left(x^{(n)}\right)\right]
$$

Le coefficient $\gamma_{-1}$ est différent de zéro, car, d'après (2) et (19), on a

et par suite

$$
w\left(x^{(n)}\right)=\sqrt[n]{\Delta_{0}\left(x^{(n)}, \omega\right)}\left(\prod_{k=1}^{n}\left|p\left(x_{k}\right)\right| e^{f\left(x_{k}\right)}\right)^{1 / n}
$$

$$
l e^{m} \sqrt[n]{\Delta_{0}\left(x^{(n)}, \omega\right)} \leqslant w\left(x^{(n)}\right) \leqslant L e^{M} \sqrt[n]{\Delta_{0}\left(x^{(n)}, \omega\right)}
$$

où $l$ et $L$ sont les bornes inférieure et supérieure de $|p(z)|$, et $m$ et $M$ celles de $f(z)$ dans l'ensemble $E$.

Remarque 1. La fonction $\Phi(z, E, w)$ a été définie par la formule (10) dans le domaine d'existence $D$ de la fonction $p(z)$. Convenons de désigner par le symbole

$$
|\Phi(z, E, \omega) p(z)|
$$

la fonction définie en dehors de $D$ par la formule

$$
|\Phi(\hat{z}, E, \omega) p(z)|=\left|\varphi\left(z, \Delta_{k}\right) p(z)\right| \quad \text { si } \quad z \in D_{k}, \quad k=\infty, 1,2, \ldots
$$

Grâce à cette convention la fonction (25) est définie dans le plan entier et il suit de ce qui précède que son logarithme est une fonction harmonique en dehors de $E^{*}$, admettant à l'infini un pôle simple, car

$$
\lim _{z \rightarrow \infty} \frac{|\Phi(z, E, \omega) p(z)|}{|z|}=\left|\gamma_{-1}\right| \text {. }
$$

Remarque 2. Si $p(z) \equiv 1$ les domaines $\Delta_{\infty}$ et $D_{\infty}$ sont identiques. Si, de plus, $f(z) \equiv 0$ et le domaine $D_{\infty}$ est simplement connexe, la fonction $w=\varphi\left(z, \Delta_{\infty}\right)$ effectue la représentation conforme de $D_{\infty}$ sur le cercle $|w|>1$ de manière que les points $z=\infty$ et $w=\infty$ se correspondent, car $\varphi\left(z, \Delta_{\infty}\right)$ est uniforme dans $D_{\infty}$, admet à l'infini un pôle simple et son module tend vers 1 lorsque $z$ tend vers la frontière de $D_{\infty}$.

4. Nouvelles limites liées aux points extrémaux. Soit $x^{(n)}=$ $=\left\{x_{0}, x_{1}, \ldots, x_{n}\right\}$ un système de points extrémaux de rang $n$ de $E$. Désignons par $\nabla\left(x^{(n)}\right)$ le produit

$$
V\left(x^{(n)}\right)=\prod_{0 \leqslant j<k \leqslant n}\left|x_{j}-x_{k}\right|,
$$

parr $v\left(x^{(n)}\right)$ et $u\left(x^{(n)}\right)$ les moyennes

$$
\begin{aligned}
& v\left(x^{(n)}\right)=\left[V\left(x^{(n)}\right)\right]^{2 / n(n+1)}, \quad n=1,2, \ldots, \\
& u\left(x^{(n)}\right)=\left(\prod_{k=1}^{n} \mid p\left(x_{k}\right) e^{f\left(x_{k}\right)} ;\right)^{1 / n}, \quad n=1,2, \ldots
\end{aligned}
$$


et par $P\left(z, x^{(n)}\right)$ le polynôme

$$
P\left(z, x^{(n)}\right)=\left(z-x_{1}\right)\left(z-x_{2}\right) \ldots\left(z-x_{n}\right) .
$$

Je dis que:

V. Les moyennes $u\left(x^{(n)}\right)$ et $v\left(x^{(n)}\right)$ oonvergent vers des limites déterminées

$$
\lim _{n \rightarrow \infty} u\left(x^{(n)}\right)=u(E, \omega), \quad \lim _{n \rightarrow \infty} v\left(x^{(n)}\right)=v_{0}(E, \omega) .
$$

Pareillement la suite $\left\{\mathfrak{n}^{\mid} \overline{\left.P\left(z, x^{(n)}\right) \mid\right\}}\right.$ converge en tout point du plan n'appartenant pas à l'ensemble $E^{*}$

$$
\lim _{n \rightarrow \infty} \sqrt[n]{\left|P\left(z, x^{(n)}\right)\right|}=P(z, E, \omega)
$$

la convergence étant uniforme dans le voisinage de tout point n'appartenant pas à $E^{*}$.

Démonstration. D'après (23) la limite

$$
\lim _{n \rightarrow \infty} w\left(x^{(n)}\right)=w(E, \omega)=1 /\left|\gamma_{-1}\right|
$$

existe et il suit de $(24)$ que $u\left(x^{(n)}\right)=w\left(x^{(n)}\right): \sqrt[n]{\Lambda_{0}\left(x^{(n)}, \omega\right)}$, donc la première des limites $(29)$ existe et on a

$$
w(E, \omega)=v(E, \omega) u(E, \omega) .
$$

Remarquons maintenant que, d'après (1) et (27),

$$
\nabla\left(x^{(n)}, \omega\right)=V\left(x^{(n)}\right)\left(\prod_{k=0}^{n}\left|p\left(x_{k}\right) e^{f\left(x_{k}\right)}\right|\right)^{-n}
$$

d'où l'on déduit la relation

$$
v\left(x^{(n)}\right)=\left[V\left(x^{(n)}, \omega\right)\right]^{2 / n(n+1)}\left[u\left(x^{(n)}\right)\right]^{2 n^{2} / n(n+1)}\left|p\left(x_{0}\right) e^{f\left(x_{0}\right)}\right|^{2 /(n+1)}
$$

dont le second membre converge vers $v(E, \omega)[u(E, \omega)]^{2}$; done la seconde des limites (29) existe et on a

$$
v_{0}(E, \omega)=v(E, \omega)[u(E, \omega)]^{2} .
$$

D'autre part, les formules (17) et (21) donnent

$$
\sqrt[n]{\left|P\left(z, x^{(n)}\right)\right|}=\sqrt[n]{\left|\Phi^{(0)}\left(z, x^{(n)}\right)\right|}|p(z)| w\left(x^{(n)}\right)
$$

d'où l'on déduit l'existence de la limite $(30)$ et la relation

$$
P(z, E, \omega)=|\Phi(z, E, \omega) p(z)| w(E, \omega)
$$

Supposons maintenant que $E$ soit la somme de deux ensembles disjoints fermés $E_{1}$ et $E_{2}$

$$
E=E_{1}+E_{2}
$$

et que parmi les points extrémaux (26) $\mu=\mu(n)$ points $x_{1}, x_{2}, \ldots, x_{\mu}$ soient situés sur $E_{1}$ et les points $x_{\mu+1}, x_{\mu+2}, \ldots, x_{n}$ sur $E_{2}$. Posons $n-\mu=$ $=\nu=\nu(n)$ et désignons par $E_{i}^{*}$ la partie de $E^{*}$ située dans $E_{i}$, $i=1,2$. Nous allons démontrer le théorème:

VI. Les suites $\{\mu(n) / n\}$ et $\{\nu(n) / n\}$ oonvergent vers des limites déterminées

(32) $\lim _{n \rightarrow \infty}[\mu(n) / n]=\alpha_{1}(E, \omega)=\alpha_{1}, \quad \lim _{n \rightarrow \infty}[v(n) / n]=\alpha_{2}(E, \omega)=\alpha_{2}$ où $\alpha_{1} \geqslant 0, \alpha_{2} \geqslant 0$ et $a_{1}+\alpha_{2}=1$.

Démonstration. Le cas où $\mu(n)=0$ ou $v(n)=0$ pour tout $n$ suffisamment grand n'exige pas de démonstration. Posons dans le cas général

$$
\begin{aligned}
& p_{n}(z)=\sqrt[\mu]{\left|\left(z-x_{1}\right) \ldots\left(z-x_{\mu}\right)\right|}, \quad n=1,2, \ldots \\
& \psi_{n}(z)=\sqrt{\left|\left(z-x_{\mu+1}\right) \ldots\left(z-x_{n}\right)\right|}, \quad n=1,2, \ldots
\end{aligned}
$$

et soit $\varphi_{n}(z)=1$ si $\mu=0$ et $\psi_{n}(z)=1$ si $y=0$. D'après $(28)$ on a indentiquement en dehors de $E$

$$
\left[\varphi_{n}(z)\right]^{\mu / n}\left[\psi_{n}(z)\right]^{\mu / n}=\sqrt[n]{\left|P\left(z, x^{(n)}\right)\right|}, \quad n_{n}=1,2, \ldots
$$

Si la suite $\mu_{n}=\mu(n) / n, n=1,2, \ldots$ n'était pas convergente, elle contiendrait deux suites partielles $\left\{\mu_{m_{k}}\right\}$ et $\left\{\mu_{n_{k}}\right\}$ tendant vers deux limites différentes $\mu_{m_{k}} \rightarrow \alpha$ et $\mu_{n_{k}} \rightarrow \bar{\alpha} \neq \alpha$. La suite $\left\{\varphi_{n}(z)\right\}$ est uniformément bornée dans tout domaine borné et, comme chaque $\varphi_{n}(z)$ est le module d'une fonction analytique en dehors des points du système $x^{(n)}$ situés dans $E_{1}$, chacune des suites $\left\{\varphi_{m_{k}}(z)\right\}$ et $\left\{\varphi_{n_{k}}(z)\right\}$ contient une suite partielle uniformément convergente ${ }^{7}$ ) en dehors de $E_{1}^{*}$. Pareillement chacune des suites $\left\{\psi_{m_{k}}(z)\right\}$ et $\left\{\psi_{n_{k}}(z)\right\}$ contient une suite partielle uniformément convergente en dehors de $E_{2}^{*}$. En changeant convenablement les suites $\left\{m_{k_{k}}\right\}$ et $\left\{n_{k}\right\}$ on peut supposer l'existence en dehors de $\boldsymbol{E}_{1}^{*}$, des limites

$$
\lim _{h \rightarrow \infty} \varphi_{m_{k}}(z)=\varphi(z), \quad \lim _{k \rightarrow \infty} q_{n_{k}}(z)=\bar{\varphi}(z)
$$

et, en dehors de $E_{2}^{*}$, des limites

$$
\lim _{k \rightarrow \infty} \psi_{m_{k}}(z)=\psi(z), \quad \lim _{k \rightarrow \infty} \psi_{n_{k}}(z)=\bar{p}(z)
$$

7) La convergence est uniforme dans le voisinage de tout point non situé dans $E_{1}^{*}$. 
et, comme le second membre de (35) converge en dehors de $E^{*}$, on a identiquemęnt

\section{et par suite}

$$
p(z)^{a} p(z)^{1-a}=\bar{p}(z) \bar{p}\left(w^{1}\right)^{1-a}
$$

$$
\bar{\alpha} \log \bar{\varphi}(z)-\alpha \log \varphi(z)=(1-\alpha) \log \psi(z)-(1-\bar{\alpha}) \log \bar{\psi}(z) .
$$

Les fonctions $\log \bar{\varphi}(z)$ et $\log \varphi(z)$ sont harmoniques en dehors de $E_{1}^{*}$ et admettent des pôles d'ordre 1 à l'infini, done le premier membre de (36) est une fonction harmonique en dehors de $\mathbb{E}_{1}^{*}$ admettant à l'infini un pôle d'ordre $\bar{a}-\alpha$. D'autre part, le second membre de (36) est harmonique en dehors de $E_{2}^{*}$ et par suite il est harmonique sur $\mathbb{E}_{1}^{*}$. On en conclut que le premier membre de (36) est harmonique dans le plan ouvert tout entier et tend vers $\infty$ si $\bar{a}>a$ ou vers $-\infty$ si $a<a$. Mais cette conclusion reste en contradiction avec le principe d'extremum. Par suite, on doit avoir $a=\bar{\alpha}$, ce qui prouve que les limites (32) existent et $\alpha_{1}+a_{2}=1$.

Remarquons que si $a=\bar{a}$ l'égalité (36) prend la forme

$$
a[\log \bar{\varphi}(z)-\log \varphi(z)]=(1-\alpha)[\log \psi(z)-\log \bar{\psi}(z)]
$$

d'où l'on conclut, par un raisonnement analogue au précédent, que les fonctions $\varphi(z)$ et $\bar{\varphi}(z)$ sont identiques. Pareillement les fonctions $\psi(z)$ êst $\bar{\psi}(z)$ doivent être identiques et par suite:

VII. Les suites (33) et (34) sont convergentes la première on dehors de $E_{1}^{*}$ et la seconde en dehors de $E_{2}^{*}$ et si l'on pose

$$
\lim _{n \rightarrow \infty} \varphi_{n}(z)=\varphi(z), \quad \lim _{n \rightarrow \infty} \psi_{n}(z)=\psi(z)
$$

on a, en dehors de $E^{*}$, la relation

$$
\varphi(z)^{\alpha} \psi(z)^{1-\alpha}=P(z, E, \omega) .
$$

Les théorèmes VI et VII peuvent être généralisés an cas où $E$ est la somme de $p \geqslant 2$ ensembles fermés disjoints

$$
E=E_{1}+E_{2}+\ldots+E_{p}
$$

et $v\left(E_{k},|z-\xi|\right)>0$ pour $k=1,2, \ldots, p$. Désignons par $\mu_{k}=\mu_{k}(n)$, $k=1,2, \ldots, p$, le nombre des points extrémanx du système $x^{(n)}$ qui sont situés sur $E_{k}$ et soient

$$
x_{n_{k-1+1}}, x_{n_{k-1}+2}, \ldots, x_{n_{k}}, \quad k=1,2, \ldots, p,
$$

où $n_{0}=-1, n_{k}=\mu_{1}+\mu_{2}+\ldots+\mu_{k}$ pour $k=1,2, \ldots, p$, les points situés sur $E_{k}$. Posons

$$
\varphi_{n, k}(z)=\sqrt[\mu_{k}]{\left|\left(z-x_{n_{k-1}+1}\right)\left(z-x_{n_{k-1}+2}\right) \ldots\left(z-n_{k}\right)\right|}, \quad l=1,2, \ldots, p
$$

si $\mu_{k}>0$ et $\varphi_{n, k}(z)=1$ si $\mu_{k}=0$.
Alors, comme dans le cas $p=2$, les limites

$$
\lim _{n \rightarrow \infty}\left[\mu_{k}(n) / n\right]=\alpha_{k}, \quad k=1,2, \ldots, p,
$$

existent et on a $a_{1}+a_{2}+\ldots+a_{p}=1$. Pareillement en dehors de l'ensemble $E_{k}^{*}=E_{l_{c}} E^{*}$ la limite

$$
\lim _{n \rightarrow \infty} q_{n, k}(z)=q_{k}(z), \quad k=1,2, \ldots, p
$$

existe et on a identiquement en dehors de $E^{*}$

$$
r_{1}(z)^{\alpha_{1}} q_{2}(z)^{\sigma_{2}} \ldots r_{p}(z)^{\alpha_{p}}=P(z, E, \omega) .
$$

Les nombres $\alpha_{k}$ et les fonctions $\psi_{k}(z)$ dépendent manifestement de l'ensemble $E$ et de la fonction génératrice $\omega$.

Faisons varier la fonction $f(z)$ sans faire varier l'ensemble $E$ et la fonction $p(z)$. La position des points extrémaux (26) dans $E$ dépend évidemment de $n$ et de $f(z)$. Pour indiquer cette dépendance nous désignerons le système $x^{(n)}$ plus exactement par

\section{Je dis que:}

$$
x^{(n, f)}=\left\{x_{0}^{(n, f)}, x_{1}^{(n, f)}, \ldots, x_{n}^{(n, f)}\right\} .
$$

VIII. Lorsqu'on ajoute ̀̀ $f(z)$ une constante quelconque c chaque système extremal $x^{(n, f)}$ reste un système extrémal $x^{(n, f+c)}$.

En effet, désignons le produit $V\left(\zeta^{(n)}, \omega\right)$ par $V\left(\zeta^{(n)}, p, f\right)$. D'après la formule (31) on a identiquement

$$
V\left(\zeta^{(n)}, p, f+c\right)=e^{-n(n+1) c} V\left(\zeta^{(n)}, p, f\right)
$$

et si $\max V\left(\zeta^{(n)}, p, f\right)=V\left(x^{(n)}, p, f\right)$ on a

$$
\max _{\left.\zeta^{(n)}\right)_{E} E}^{\zeta^{(n)} E} V\left(\zeta^{(n)}, p, f+c\right)=e^{-n(n+1) c} V\left(x^{(n)}, p, f\right)=V\left(x^{(n)}, p, f+c\right)
$$

ce qui prouve notre thèse.

5. Distribution des points extrémaux dans quelques cas particuliers. Supposons que $p(z) \equiv 1$, que $E$ soit la somme d'un nombre fini $p$ continus disjoints $F_{1}, F_{2}, \ldots, F_{p}$ (fig. 1.)

(37) $\quad E=T_{1}+H_{2}+\ldots+F_{p}$ et que $f(z)$ se réduise sur chaque $F_{k}$ à une constante $\lambda_{k}$, $f(z)=\lambda_{k} \quad$ si $\quad z \in F_{k} ; \quad k=1,2, \ldots, p$.

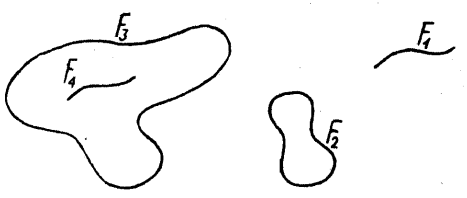
Fig. 1

Une telle fonction sera dite fonction en escalier. Supposons encore que chaque $F_{k}$ se réduise à la frontière du domaine $D_{\infty}\left(F_{k}\right)$. 
Alors le domaine $D$ est le plan entier, la fonction

$$
\log \Phi(z, E, 1, f)
$$

est harmonique partout en dehors de $E^{*}$ et du point $z=\infty$, reste continue dans $E^{*}$ et admet la valeur $\lambda_{k}$ aux points de l'ensemble

$$
F_{k}^{*}=E^{*} F_{k}, \quad k=1,2, \ldots, p .
$$

A l'infini $\log \Phi(z, E, 1, f)$ a un pôle d'ordre 1. D'après (19) et (20)

(39) $w\left(x^{(n)}\right)=\sqrt[n]{\Delta_{0}\left(x^{(n)}, \omega\right)} e^{h_{n}} \quad$ où $\quad h_{n}=\frac{f\left(x_{1}\right)+f\left(x_{2}\right)+\ldots+f\left(x_{n}\right)}{n}$

et on a vu que les limites

$$
\lim _{n \rightarrow \infty} h_{n}=h, \quad \lim _{h \rightarrow \infty} w\left(x^{(n)}\right)=v(E, 1, f) e^{h}
$$

existent et que

$$
\lim _{z \rightarrow \infty} \frac{\Phi(z, E, 1, f)}{|z|}=\frac{1}{v(E, 1, f) e^{h}}
$$

Nous allons examiner la répartition de l'ensemble $E^{*}$ sur les continus $F_{1}, F_{2}, \ldots, F_{p}$. Pour cela désignons par $G\left(z, F_{k}\right)$ la fonction de Green du domaine $D_{\infty}\left(F_{k}\right)$ de pôle à l'infini. On a vu dans le numére 2 que $G\left(z, F_{k}\right)=\log \Phi\left(z, F_{k}, 1,0\right)$ d'où il suit que

$$
\log \Phi\left(z, F_{k}, 1, f\right)=G\left(z, F_{k}\right)+\lambda_{k} \text {. }
$$

IX. Si $\lambda_{1}<\min \left(\lambda_{2}, \lambda_{3}, \ldots, \lambda_{p}\right)$ la partie $F_{1}$ de $E$ est entièrement couverte par $E^{*}$, e'est-à-dire $F_{1}^{*}=F_{1}$. Pareillement $F_{1}^{*}=F_{1}$ si $\lambda_{1} \leqslant$ $\leqslant \min \left(\lambda_{2}, \lambda_{3}, \ldots, \lambda_{p}\right)$ et $F_{1}$ est situé sur la frontière du domaine $\left.D_{\infty}(E)^{8}\right)$.

Démonstration. D'après (14) la fonction (38) satisfait sur $F_{1}$ à l'inégalité $\log \Phi(z, E, 1, f) \leqslant \lambda_{1}$ et d'après (15) elle satisfait partout à la suivante: $\log \Phi(z, E, 1, f) \geqslant \lambda_{1}$. Si un point $z_{0}$ de $F_{1}$ n'appartenait pas à $F_{1}^{*}$ la fonction (38) serait harmonique en ce point et $y$ atteindrait son minimum $=\lambda_{1}$ sans être constante dans le voisinage de $z_{0}$, ce qui reste en contradiction avec le principe du minimum.

Pour prouver que la fonction (38) n'est pas constante dans le roisinage de $z_{0}$ désignons par $\Delta$ le domaine partiel de $\Pi-E^{*}$ contenant $z_{0}$. Si $\Delta$ est borné la frontière de $\Delta$ contient des points appartenant à l'ensemble $F_{2}^{*}+F_{3}^{*}+\ldots+F_{p}^{*}$ et en ces points $\log \Phi(z, E, 1, f)>\lambda_{1}$ car alors $\lambda_{1}<\min \left(\lambda_{2}, \lambda_{3}, \ldots, \lambda_{p}\right)$. D'autre part, si $\Delta$ n'est pas borné la fonction (38) n'est pas constante dans $\Delta$, car elle tend vers l'infini avec $\approx$.

8) Si le continu $F_{1}$ ne se réduit pas à la frontière du domaine $D_{\infty}\left(F_{1}\right)$, par exemple s'il possède des points intérieurs, le théorème reste vrai lorsqu'on remplace, dans son énoncé, $F_{1}$ par la frontière de $D_{\infty}\left(F_{1}\right)$.
X. Si pour toutes les valeurs de $k>1$

$$
\lambda_{k}>\lambda_{1}+\max _{z_{6} F_{k}} G\left(z, F_{1}\right), \quad k=2,3, \ldots, p,
$$

l'ensemble $E^{*}$ se réduit d̀ $F_{1}$ et par suite l'ensemble $\sum_{k=2}^{p} F_{k}^{*}$ est vide; si pour au moins une valeur de $k$

$$
\lambda_{k}<\lambda_{1}+\max _{z_{\theta} F_{k}} G\left(z, F_{1}\right)
$$

l'ensemble $\sum_{k=2}^{p} F_{k}^{*}$ n'est pas vide.

Démonstration. $1^{\circ}$ Supposons que les inégalités (41) soient satisfaites. Alors $E^{*} \supset F_{1}$ d'après le théorème IX. Posons $\sum_{k=2}^{p} F_{k}^{*}=A+B$ où $A$ est la partie de $\sum_{k=2}^{p} F_{k}^{*}$ située dans le domaine $D_{\infty}\left(F_{i_{1}}\right)$ et $B=\sum_{k=2}^{p} F_{k}^{*}-A$. La différence

$$
r(z)=\log \Phi(z, E, 1, f)-G\left(z, F_{1}\right)
$$

est harmonique dans le domaine $D_{\infty}\left(F_{1}\right)-A$, le point à l'infini y compris, et continue sur la frontière $F_{1}+A$ de ce domaine. Comme $F_{1}^{*}=F^{*}$ on a $r(z)=\lambda_{1}$ sur $F_{1}$. D'après (39) et $(40) h_{n} \geqslant \lambda_{1}$ car $\lambda_{k} \geqslant \lambda_{1}$ et par suite $h \geqslant \lambda_{1}$. D'autre part

done

$$
v(E, 1, f) \geqslant v\left(F_{1}, 1, f\right)=v\left(F_{1},|z-\zeta|\right) e^{-2 \lambda_{1}}
$$

$$
1 /\left[v(E, 1, f) e^{h}\right] \leqslant e^{\lambda_{1}} / v\left(\boldsymbol{F}_{1},|z-\zeta|\right)
$$

et comme la valeur de $r(z)$ à l'infini

$$
r(\infty)=\log \frac{1}{v(E, 1, f) e^{h}}-\log \frac{1}{v\left(F_{1},|z-\zeta|\right)}
$$

on a $r(\infty) \leqslant \lambda_{1}$.

Il s'ensuit que l'ensemble $A$ doit être vide, car dans le cas contraire on aurait sur la partie $F_{k}^{*}$ de $A$

$$
r(z)=\lambda_{k}-G\left(z, F_{1}\right) \geqslant \lambda_{k}-\max _{z_{G} F_{k}} G\left(z, F_{1}\right)>\lambda_{1}
$$

ce qui est impossible d'après le principe du minimum, vu l'inégalité $r(\infty) \leqslant \lambda_{1}$. D'autre part, l'ensemble $B$ est aussi vide car, d'après le prin$r(\infty) \leqslant \lambda_{1}$. D'autre part, lux es points extrémaux sont situés sur $F_{1}$. Par suite $E^{*}$ se réduit à $F_{1}$. 
$2^{\circ}$ Supposons maintenant que l'inégalité (42) soit satisfaite pour $k=\alpha$ et soit $z_{0}$ un point de $F_{\alpha}$ en lequel $G\left(z_{0}, F_{1}\right)=\max _{z_{G} F_{\alpha}^{\prime}} G\left(z, F_{1}\right)$. Si l'ensemble $\sum_{k=2}^{n} F_{k}^{*}$ était vide on aurait identiquement

$$
\Phi(z, E, 1, f)=\Phi\left(z, F_{1}, 1, f\right)
$$

et comme $\log \Phi\left(z, F_{1}, 1,0\right)=G\left(z, F_{1}\right)$ et $f(z)=\lambda_{1}$ sur $F_{1}$ on aurait $\log \Phi(z, E, 1, f)=G\left(z, F_{1}\right)+\lambda_{1}$ et la différence (43) se réduirait à la constante $\lambda_{1}$, ce qui est impossible car

$$
r\left(z_{0}\right) \leqslant \lambda_{a}-G\left(z_{0}, F_{1}\right)=\lambda_{\alpha}-\max _{z_{e} F_{a}} G\left(\approx, F_{1}\right)<\lambda_{1} .
$$

Le théorème est donc démontré.

Supposons maintenant que l'ensemble (37) se réduise à la frontière du domaine $D_{\infty}(E)$ et désignons par $G(z, E)$ la fonction de Green de ce domaine de pôle à l'infini. On sait que l'équation $G(z, E)=\mu$ où $\mu$ est un nombre positif quelconque, définit une courbe $\Gamma_{\mu}$ composée d'un seul ou de plusieurs contours $C_{1}, C_{2}, \ldots, O_{q}$ où $1 \leqslant q \leqslant p$ tels que chaque continu $F_{k}$ est situé dans l'intérieur de l'un des contours $C_{1}, C_{2}, \ldots, C_{q}$ et que chaque $C_{k}$ contient dans son intérieur au moins un des continus $F_{1}, F_{2}, \ldots, F_{p}$. Les intérieurs de $C_{i}$ et $C_{k}$, où $i \neq k$, sont toujours disjoints. Si la constante $\mu$ est suffisamment grande le nombre $q$ est égal à 1 et si $\mu$ est suffisamment petite on a $q=p$, et alors chaque $F_{k}$ est entouré par un et un seul des contours $C_{1}, C_{2}, \ldots, O_{p}$. Ceci posé nous allons démontrer le théorème:

$\mathrm{XI}$. Si l'ensemble $E=F_{1}+F_{2}+\ldots+F_{p}$ se réduit à la frontière $d u$ domaine $D_{\infty}(E)$ et $\lambda_{1}=\min \left(\lambda_{1}, \lambda_{2}, \ldots, \lambda_{p}\right)$ alors $\dot{a}$ chaque $k=2,3, \ldots, p$ correspond un nombre $\mu_{k}>0$ tel que si

$$
\lambda_{k}<\mu_{k}+\lambda_{1}
$$

le continu $F_{k}$ est entièrement couvert par $F_{k}^{*}$ et par suite

$$
\log \Phi(z, E, 1, f)=\lambda_{k} \quad \text { pour } \quad z \in F_{k} .
$$

Démonstration. On a vu que $F_{1}^{*}=F_{1}$. Soit $\mu_{k}$ un nombre positif tel que la courbe $G(z, E)=\mu_{k}$ contienne un contour $O_{k}$ entourant le continu $F_{k}$ et n'entourant aucun des continus $F_{1}, F_{2}, \ldots, F_{k-1}, F_{k+1}, \ldots$,
$F_{p}$. La différence

$$
R(z)=\log \Phi(z, E, 1, f)-G(z, E)
$$

est harmonique dans le domaine $D_{\infty}(E)$, le point $z=\infty \mathrm{y}$ compris, et satisfait sur la frontière $E$ de ce domaine à l'inégalité $R(z) \geqslant \lambda_{1}$ car d'après (15) $\log \Phi(z, E, 1, f) \geqslant \lambda_{1}$ sur $E$. Par suite $R(z) \geqslant \lambda_{1}$ dans $D_{\infty}(E)$ et

$$
\log \Phi(z, E, 1, f) \geqslant \mu_{k}+\lambda_{1} \quad \text { sur } \quad C_{k} .
$$

Soit $G_{k}$ le domaine limité par $C_{k}$ et $F_{k}^{*}$. Le $\log \Phi(z, E, 1, f)$ est harmonique dans $G_{k}$, continu sur la fermeture de ce domaine et admet sur $F_{k}^{*}$ la valeur $\lambda_{k}$ et sur $C_{k}$ des valeurs $\geqslant \mu_{k}+\lambda_{1}>\lambda_{k}$ done

$$
\log \Phi(z, E, 1, f)>\lambda_{k} \quad \text { dans } \quad G_{k} .
$$

Si un point $z_{0}$ de $F_{k}$ n'appartenait pas à $F_{k}^{*}$ il appartiendrait à $G_{k}$, ce qui est impossible d'après le principe du minimum, car sur $F_{k}$ on a $\log \Phi(z, E, 1, f) \leqslant \lambda_{k}$. Par suite chaque point de $F_{k}$ est couvert par $F_{k}^{*}$ et l'égalité (44) est démontrée.

Corollarre. Si l'ensemble $E=F_{1}+F_{2}+\ldots+F_{p}$ se réduit à la frontière du domaine $D_{\infty}(E), f(z)=\lambda_{k}$ sur $F_{k}$ pour $k=1,2, \ldots, p$ et la différence

$$
\max \left(\lambda_{1}, \lambda_{2}, \ldots, \lambda_{p}\right)-\min \left(\lambda_{1}, \lambda_{2}, \ldots, \lambda_{p}\right)
$$

est suffisamment petite, l'ensemble $E$ est entièrement couvert par $E^{*}$ et $\log \Phi(z, E, 1, f)$ est égal d $f(z)$ ti tout point de $E$.

Soit $F_{0}$ la frontière commune d'un domaine borné simplement connexe $G$ et du domaine non borné $D_{\infty}\left(F_{0}\right), F_{1}$ un ensemble fermé contenu dans $G$ (fig. 2) et $f(z)$ la fonction égale à $\lambda_{0}$ sur $F_{0}$ et à $\lambda_{1}$ sur $F_{1}$, où $\lambda_{0}$ et $\lambda_{1}$ sont des constantes réelles quelconques. Il est clair que l'écart $v(E, 1, f)$, où $E=F_{0}+F_{1}$ n'est pas plus petit que les écarts

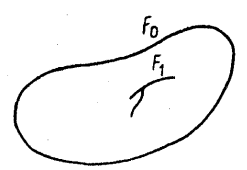

Fig. 2 $v\left(F_{0}, 1, f\right)$ et $v\left(F_{1}, 1, f\right)$. Je dis que:

XII. Si $\lambda_{0}>\lambda_{1}$ on $a v(E, 1, f)>v\left(F_{0}, 1, f\right)$ et $s i$

$$
\lambda_{0}<\lambda_{1}+\log \left(v_{0} / v_{1}\right),
$$

où $v_{i}=v\left(F_{i},|\hat{z}-\zeta|\right), i=0,1$, on a aussi $v(E, 1, f)>v\left(F_{1}, 1, f\right)$.

Démonstration. Soit $x^{(n)}=\left\{x_{0}, x_{1}, \ldots, x_{n}\right\}$ un soystème de points extrémaux de rang $n$ de $E$ par rapport à $\omega=|z-\zeta| \exp [-f(z)-f(\zeta)]$ et $\eta^{(n)}=\left\{\eta_{0}, \eta_{1}, \ldots, \eta_{n}\right\}$ un système de $n=\mu+\nu$ points de $E$ dont les points $\eta^{(\mu)}=\left\{\eta_{0}, \eta_{1}, \ldots, \eta_{\mu}\right\}$ sont situés sur $F_{0}$ et les points $\eta^{(\nu-1)}=$ $=\left\{\eta_{\mu+1}, \ldots, \eta_{n}\right\}$ sur $F_{1}$. Il est clair que $V\left(x^{(n)}, \omega\right) \geqslant V\left(\eta^{(n)}, \omega\right)$. En désignant par $V\left(\eta^{(n)}\right)$ le produit

$$
V\left(\eta^{(n)}\right)=\prod_{0 \leqslant i<k \leqslant n}\left|\eta_{i i}-\eta_{k}\right|
$$

on a l'inégalité

$$
V\left(x^{(n)},(1)\right) \geqslant \frac{V\left(\eta^{(n)}\right)}{\exp \left\{n\left[(\mu+1) \lambda_{0}+v \lambda_{1}\right]\right\}} .
$$


Supposons que les systèmes $\eta^{(\mu)}$ et $\eta^{(\nu-1)}$ soient extrémaux respectivement sur $F_{0}$ et $F_{1}$ par rapport à la distance $|z-\zeta|$ et que les nombres $\mu$ et $\nu$ croissent avec $n$ de manière que lorsque $n \rightarrow \infty$

$$
\mu / n \rightarrow 1-\alpha, \quad \nu / n \rightarrow \alpha,
$$

où $a$ est un nombre de l'intervalle $0<\alpha<1$. Alors les moyennes

$$
v_{n}^{(0)}=\left[V\left(\eta^{\mu}\right)\right]^{2 / \mu(\mu+1)}, \quad \eta_{n}^{(1)}=\left[V\left(\eta^{\mu-1}\right)\right]^{2 / \nu(\nu-1)}, \quad \mu=1,2, \ldots
$$

convergent respectivement vers $v_{0}=v\left(F_{0},|z-\zeta|\right)$ et $v_{1}=v\left(F_{1},|z-\zeta|\right)$ et la suite

$$
I_{\mu}(z)=\left|\left(z-\eta_{0}\right)\left(z-\eta_{1}\right) \ldots\left(z-\eta_{\mu}\right)\right|^{1 /(\mu+1)}
$$

converge dans le domaine $G$ vers la constante $v_{0}$, la convergence étant uniforme dans le voisinage de tout point de $G$ (cf. [2]). Mais on a identiquement

$$
\nabla\left(\eta^{(n)}\right)=\nabla\left(\eta^{(\mu)}\right) V\left(\eta^{(\mu-1)}\right) \cdot I_{n}^{(\mu+1) r}
$$

où $I_{n}=\left[\prod_{k=\mu+1}^{n} I_{\mu}\left(\eta_{k}\right)\right]^{1 / v} \rightarrow v_{0}$ lorsque $n \rightarrow \infty$ et, d'après $(46)$ où

$$
\left[V\left(x^{(n)}, \omega\right)\right]^{2 / n(n+1)} \geqslant v_{n}^{(0) \mu(\mu+1) / n(n+1)} v_{n}^{(1) \nu(\nu-1) / n(n+1)} I_{n}^{2(\mu+1) \nu / n(n+1)} e^{-h_{h}},
$$

$$
l_{n}=2\left[\frac{\mu+1}{n+1} \lambda_{0}+\frac{\nu}{n+1} \lambda_{1}\right] .
$$

En faisant tendre $n$ vers l'infini on en déduit l'inégalité

$$
v(E, \omega) \geqslant v_{0}^{(1-\alpha)^{2}} v_{1}^{\alpha^{2}} v_{0}^{2 \alpha(1-\alpha)} \exp \left\{-2\left[(1-\alpha) \lambda_{0}+\alpha \lambda_{1}\right]\right\}
$$

dont le second membre est égal à

$$
\left(v_{0} / e^{2 \lambda_{0}}\right)\left[\left(v_{1} / v_{0}\right)^{\alpha} e^{2 \lambda_{0}} / e^{2 \lambda_{1}}\right]^{\alpha}
$$

et comme $v\left(F_{0}, 1, f\right)=v_{0}, e^{-2 \lambda_{0}}$ et l'expression entre parenthèses [ ] surpasse 1 si $\lambda_{0}>\lambda_{1}$ et $\alpha$ est suffisamment petit on voit que $v(E, 1, f)>$ $>v\left(F_{0}, 1, f\right)$ si $\lambda_{0}>\lambda_{1}$. Le second membre de (4.7), où l'on a remplacé $1-\alpha$ par $\beta$, prend la forme

$$
\left(v_{1} / e^{2 \lambda_{1}}\right)\left[\left(v_{0} / r_{1}\right)^{2-\beta} e^{2 \lambda_{1}} / e^{2 \lambda_{0}}\right]^{\beta},
$$

où l'expression entre parenthèses [ ] surpasse 1 poưr $\beta=0$ si $\lambda_{0}$ satisfait à l'inégalité (45). Comme $v\left(F_{1}, 1, f\right)=v_{1} e^{2 \lambda_{1}}$ on a $v(E, 1, f)>v\left(F_{1}, 1, f\right)$, si la condition (45) est remplie et par suite la thèse est démontrée.

Supposons maintenant que $E$ soit la somme de $p+1$ continus disjoints $E=F_{0}+F_{1}+\ldots+F_{p}$, constituant la frontière d'un domaine borné $D(E)$ dont la frontière extérieure est $F_{0}$ et la frontière intérieure est $F=F_{1}+F_{2}+\ldots+F_{p}$ (fig. 3 ). Supposons encore que $F_{0}$ soit en même temps la frontière du domaine $D_{\infty}(E)$ et que

$$
f(z)=\lambda_{k} \quad \text { pour } \quad z \in F_{k}, \quad k=0,1, \ldots, p .
$$

Formons la fonction de Green $G\left(z, F_{0}\right)$ du domaine $D_{\infty}(E)$ de pôle à l'infini, la fonction $\Phi(z, F, 1, f)$, et posons

$$
M_{0}=\max _{z_{\in} F_{0}} \log \Phi(z, F, 1, f)
$$

XIII. $1^{\circ}$ Si $\lambda_{0} \leqslant \min \left(\lambda_{1}, \lambda_{2}, \ldots, \lambda_{p}\right)$ l'ensemble $E^{*}$ se réduit à $F_{0}$ et la fonction $\log \Phi(z, E, 1, f)$ est égale d̀ $G\left(z, F_{0}\right)+\lambda_{0}$ dans le domaine $D_{\infty}(E)$ et $\grave{a} \lambda_{0}$ en dehors de ce domaine.

$2^{\circ}$ Si $\lambda_{0}>M_{0}$ l'ensemble $F_{0}^{*}$ est vide

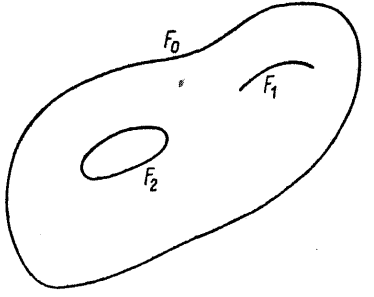

Fig. 3 et $\Phi(z, E, 1, f) \equiv \Phi(z, F, 1, f)$.

$3^{\circ}$ Si $\lambda_{0}<M_{0}$, l'ensemble $F_{0}^{*}$ n'est pas vide.

$4^{\circ}$ Si $\lambda_{0}>\min \left(\lambda_{1}, \lambda_{2}, \ldots, \lambda_{p}\right)$, l'ensemble $\sum_{k=1}^{p} F_{k}^{*} n^{\prime}$ est pas vide.

Démonstration. $1^{0}$ Soit $\lambda_{0} \leqslant \min \left(\lambda_{1}, \lambda_{2}, \ldots, \lambda_{p}\right)$. Alors

$$
V\left(\zeta^{(n)}, \omega\right)=\frac{V\left(\zeta^{(n)}\right)}{\exp \left\{n\left[f\left(\zeta_{0}\right)+\ldots+f\left(\zeta_{n}\right)\right]\right\}} \leqslant \frac{V\left(\zeta^{(n)}\right)}{\exp \left[n(n+1) \lambda_{0}\right]}
$$

et par suite

$$
\max _{\zeta^{(n)} \in E} V\left(\zeta^{(n)}, \omega\right) \leqslant \max _{\zeta^{(n)} \in E} V\left(\zeta^{(n)}\right) \exp \left[-n(n+1) \lambda_{0}\right]
$$

Mais, comme d'après le principe du maximum, $\max _{\zeta^{(n)} \in E} V\left(\zeta^{(n)}\right)=$ $=\max _{\left.\zeta^{(n)}\right)} V\left(\zeta^{(n)}\right)$, on a

$$
\max _{\zeta^{(n)} \in E} V\left(\zeta^{(n)}, \omega\right) \leqslant \max _{\zeta^{(n)} \in F_{0}} V\left(\zeta^{(n)}\right) e^{-n(n+1) \lambda_{0}}=\max _{\zeta^{(n)} F_{F} F_{0}} V\left(\zeta^{(n)}, \omega\right)
$$

et il est clair que

$$
\max _{\zeta^{(n)} \in E} V\left(\zeta^{(n)}, \omega\right) \geqslant \max _{\zeta^{(n)} \in F_{0}} V\left(\zeta^{\zeta(n)}, \omega\right)
$$

donc les points extrémaux sm: zitués sur $F_{0}$ et par suite $E^{*} \subset F_{0}$.

D'autre part, la fonction $\left.)_{1} \cdots / \%, E, 1, f\right)$ est harmonique en dehors de $E^{*}$ et satisfait partout à $1 . \quad$ ité $\log \Phi(z, E, 1, f) \geqslant \lambda_{0}$ et sur $F_{0}$ à la suivante $\log \Phi(z, E, 1, f) \leqslant \lambda_{0}$. ul un point $z_{0}$ de $F_{0}$ n'appartenait pas à $E^{*}$ la fonction serait harmonique en $z_{0}$ et $y$ atteindrait son minimum sans être constante dans le voisinage de $z_{0}$, ce qui est impossible, donc $E^{*} \supset F_{0}$ et par suite $E^{*}=F_{0}$. 
Il en résulte que $\log \Phi(z, E, 1, f)=\lambda_{0}$ sur $F_{0}$ et en dehors du domaine $D_{\infty}(E)$. La différence $\log \Phi(z, E, 1, f)-\lambda_{0}$ est égale à $G\left(z, F_{0}\right)$ dans $D_{\infty}\left(F_{0}\right)$ car elle s'annule sur la frontière de ce domaine et admet un pôle à l'infini.

$2^{\circ}$ Soit $\lambda_{0}>M_{0}$. Si l'ensemble $F_{0}^{*}$ n'était pas vide on aurait aux points de $F_{0}^{*}$

$$
\Phi(z, E, 1, f)=e^{\lambda_{0}}>e^{M_{0}} \geqslant \Phi(\varepsilon, F, 1, f)
$$

ce qui est impossible, car d'après (13) on a partout;

$$
\Phi(z, E, 1, f) \leqslant \Phi(z, F, 1, f) .
$$

$3^{\circ}$ Si $\lambda_{0}<M_{0}$ l'ensemble $F_{0}^{*}$ ne peut être vide, car dans le cas contraire on aurait partout $\Phi(z, E, 1, f)=\Phi(z, F, 1, f)$ et, si $z_{0}$ est un point de $F_{0}$ où $\log \Phi\left(z_{0}, F, 1, f\right)=M_{0}$, on a

$$
\log \Phi\left(z_{0}, E, 1, f\right) \leqslant \lambda_{0}<M_{0}=\log \Phi\left(z_{0}, \dot{F}, 1, f\right)
$$

et les fonctions $\Phi(z, E, 1, f)$ et $\Phi\left(z, F^{1}, 1, f\right)$ ne sont pas identiques.

$4^{0}$ Si $\lambda_{0}>\min \left(\lambda_{1}, \lambda_{2}, \ldots, \lambda_{p}\right)$, l'ensemble $\sum_{k=1}^{p} F_{l_{i}}^{*}$ n'est pas vide, car d'après le théorème IX l'ensemble $F_{a}^{*}$ esti identique à $F_{a}$, si $\lambda_{a}=$ $=\min \left(\lambda_{0}, \lambda_{1}, \ldots, \lambda_{p}\right)$.

6. Quelques applications. A. Soit; $E$ la somme de $p$ continus disjoints $E=F_{1}+F_{2}+\ldots+F_{p}$ constituant la frontière du domaine $D_{\infty}(E)$. On sait que $\log \Phi(z, E, 1,0)$ est la fonction de Green du domaine $D_{\infty}(E)$ de pôle à l'infini. Désignons par $\varphi_{k}(z)$ la fonction égale à 1 sur $F_{k}$ et à zéro sur $E-F_{k}$. La différence

$$
\log \Phi\left(z, E, 1, \lambda r_{k}\right)-\log \Phi(z, E, 1,0)
$$

cù $\lambda$ est un paramètre réel $\neq 0$, est harmonique dans $D_{\infty}(\boldsymbol{E})$ le point $z=\infty$ y compris, s'annule sur $E-F_{k}$ et, si $\lambda$ est suffisamment petit, elle est égale à $\lambda$ sur $F_{k}$. Par suite, lorsque $\lambda$ est suffisamment petit, l'expression

$$
\frac{1}{\lambda} \log \frac{\Phi\left(z, E, 1, \lambda \varphi_{k}\right)}{\Phi(z, E, 1,0)}
$$

est la mesure harmonique du continu $F_{k}$ par rapport an domaine $D_{\infty}(E)$.

Plus généralement, soit $f(z)$ une fonction réelle continue quelconque définie sur $E$. Formons l'expression

$$
\frac{1}{\lambda} \log \frac{\Phi(z, E, 1, \lambda f)}{\Phi(z, E, 1,0)}
$$

où $\lambda$ est un paramètre réel $\neq 0$. Si, pour une valeur suffisamment petite de $\lambda, \log \Phi(z, E, 1, \lambda f)=\lambda f$ sur $E$ (ce qui a toujours lieu lorsque $f(z)$ est une fonction en escalier), alors l'expression (48) est la solution du problème de Dirichlet pour le domaine $D_{\infty}(E)$ et les données frontières $f(z)$.

B. Considérons le cas particulier $p=2, E=F_{1}+F_{2}$ et désignons par $\varphi\left(z, D_{\infty}, 1, \lambda \varphi_{1}\right)$ la fonction holomorphe dans le domaine $D_{\infty}(E)$ de module $\Phi\left(z, E, 1, \lambda \varphi_{1}\right)$. Par définition, $\varphi_{1}(z)=1$ sur $F_{1}$ et $\varphi_{1}(z)=0$ sur $F_{2}$. L'expression

$$
\psi(z)=\left[\frac{\varphi\left(z, D_{\infty}, 1, \lambda \varphi_{1}\right)}{\varphi\left(z, D_{\infty}, 1,0\right)}\right]^{1 / \lambda}
$$

est une fonction holomorphe (en général multiforme) dans le domaine doublement connexe $D_{\infty}(E)$ contenant le point $z=\infty$. Le module de $\psi(z)$ est continu dans $D_{\infty}(E)+E$ et il est égal à 1 sur $F_{2}$. Si $\lambda>0$ est suffisamment petit, on a $|\psi(z)|=e$ sur $F_{1}$.

Lorsque $z$ parcourt un contour $C_{1}$ entourant $F_{1}$ et n'entourant pas $F_{2}, \psi(z)$ se multiplie par un facteur $e^{2 \pi i \alpha}$, où $0<a<1$, et par suite $\psi(z)^{1 / \alpha}$ se multiplie par $~^{2 \pi i}$. Il en résulte que, lorsque $z$ parcourt un contour $C_{2}$ entourant $F_{3}, \psi(z)^{1 / a}$ se multiplie par $e^{-2 \pi i}$, d'où l'on conclut que la fonction $w=\psi(z)^{1 / a}$ effectue la représentation conforme du domaine $D_{\infty}(E)$ sur une couronne circulaire.

C. Considérons le cas général où $E=F_{1}+F_{2}+\ldots+F_{p}$. Soit $x^{(n, f)}=$ $=\left\{x_{0}^{(n, f)}, x_{1}^{(n, f)}, \ldots, x_{n}^{(n, f)}\right\}$ un système des points extrémaux du rang $n$ de $E$ par rapport à la fonction $\omega=|z-\zeta| \exp [-f(z)-f(\zeta)]$ et $\mu_{k}^{(n, f)}$ le nombre des points du système $x^{(n, f)}$ qui sont situés sur $F_{k}, k=1,2, \ldots, p$. On sait que la limite

$$
\lim _{n \rightarrow \infty} \frac{\mu_{k}^{(n, f)}}{n}=\alpha_{k}(f), \quad k=1,2, \ldots, p,
$$

existe et que les fonctionnelles $\alpha_{k}(f)$ satisfont aux relations

$$
0 \leqslant \alpha_{k}(f) \leqslant 1, \quad \sum_{1}^{p} \alpha_{k}(f)=1
$$

D'autre part, il s'ensuit du théorème VIII que, si $c$ est une constante, on a $\alpha_{k}(f+c)=\alpha_{k}(f), k=1,2, \ldots, p$.

Supposons que $f(z)$ soit une fonction en escalier. Nous écrirons

$$
f=\left(\lambda_{1}, \lambda_{2}, \ldots, \lambda_{p}\right),
$$

si $f(z)=\lambda_{k}$ sur $F_{k}, k=1,2, \ldots, p$. Les fonctionnelles $\alpha_{k}(f)$ sont alors des fonctions de $p$ variables $\lambda_{1}, \lambda_{2}, \ldots, \lambda_{p}$ définies pour toutes les valeurs réelles de ces variables. Désignons, comme plus haut, par $G\left(z, F_{k}\right)$ la fonction égale dans le domaine $D_{\infty}\left(F_{k}\right)$ à la fonction de Green de ce domaine de pôle à l'infini et égale à zéro en dehors de $D_{\infty}\left(F_{k}\right)$. 
XIV. Les fonctions $\alpha_{k}(f), \eta_{k}=1,2, \ldots, p$, jouissent des proprietes suivantes:

$1^{\circ} S i, k$ étant fixe quelconque,

$\lambda_{k} \leqslant \lambda_{j}-\max _{z_{e} F_{j}} G\left(z, F_{k}\right) \quad$ pour tout $\quad j \neq k, \quad$ on $a \quad \alpha_{k}(f)=1$

$\lambda_{k}>\lambda_{j}+\max _{z_{G}} G\left(z, F_{j}\right) \quad$ pour un $j \neq k, \quad$ on $a \quad \alpha_{k}(f)=0$

$2^{\circ}$ Quelles que soient $f=\left(\lambda_{1}, \lambda_{2}, \ldots, \lambda_{p}\right)$ et $f^{\prime}=\left(\gamma_{1}^{\prime}, \lambda_{2}^{\prime}, \ldots, \lambda_{p}^{\prime}\right)$ on a l'inégalité

$$
\sum_{k=1}^{p}\left(\lambda_{k}^{\prime}-\lambda_{k_{k}}\right)\left[{ }^{\prime} k_{k}\left(f^{\prime}\right)-a_{k}(f)\right] \leqslant 0 .
$$

Démonstration. $1^{0}$ Si $\lambda_{k} \leqslant \lambda_{j}-\max _{F_{i}} G\left(z, F_{k}\right)$ pour tout $j \neq k$, il résulte du théorème $X$ que $E^{*}=E_{k}$ et par suite $\alpha_{k}(f)=1$. Supposons qu'on ait

$$
\lambda_{k}>\lambda_{a}+\max _{F_{k}} G\left(z, F_{\alpha}\right),
$$

où $a \neq k$ est fixe. Si l'ensemble $F_{k}^{*}$ n'était pas vide, on aurait en tout point $z_{0} \in F^{*}$

$$
\lambda_{k}=\log \Phi\left(z_{0}, E, 1, f\right) \leqslant \log \Phi\left(z_{0}, F_{a}, 1, f\right),
$$

car on a, quel que soit $z, \Phi(z, E, 1, f) \leqslant \Phi\left(z, F_{a}, 1, f\right)$ et, comme $\log \Phi\left(z, F_{\alpha} 1, f\right)=\lambda_{\alpha}+G\left(\hat{z}, F_{\alpha}\right)$, on aurait

$$
\lambda_{k} \leqslant \lambda_{\alpha}+G\left(z_{0}, F_{\alpha}\right) \leqslant \lambda_{\alpha}+\max G^{*}\left(z, F_{a}\right),
$$

ce qui reste en contradiction avec l'hypothèse. Par suite l'ensemble $F_{k}^{*}$ est vide et $\alpha_{k}(f)=0$.

$2^{\circ}$ Soient $x^{(n, f)}$ et $x^{\left(n, f^{\prime}\right)}$ deux systèmes de points extrémaux de $E$ correspondant respectivement à $\omega=|z-\zeta| \exp [-f(z)-f(\zeta)]$ et $\omega^{\prime}=$ $=|z-\zeta| \exp \left[-f^{\prime}(z)-f^{\prime}(\zeta)\right]$. Alors

$$
\begin{aligned}
V\left(x^{(n, f)}, \omega\right) & =V\left(x^{(n, f)}\right) \exp \left[-n \sum \lambda_{k} \mu_{k}(n, f)\right] \\
& \geqslant V\left(x^{\left(n, f^{\prime \prime}\right)}\right) \exp \left[-n \sum \lambda_{k} \mu_{k}\left(n, f^{\prime}\right)\right], \\
V\left(x^{\left(n, f^{\prime}\right)}, \omega^{\prime}\right) & =V\left(x^{\left(n, f^{\prime}\right)}\right) \exp \left[-n \sum \lambda_{k}^{\prime} \mu_{k}\left(n, f^{\prime}\right)\right] \\
& \geqslant V\left(x^{(n, f)}\right) \exp \left[-n \sum \lambda_{k}^{\prime} \mu_{k}(n, f)\right]
\end{aligned}
$$

où la somme $\sum$ est étendue aux valeurs $1,2, \ldots, p$ de $k$. Puisque

on

$$
\left[\nabla\left(x^{(n, f)}, \omega\right)\right]^{2 / n(n+1)} \rightarrow v(E, \omega), \quad\left[V\left(x^{(n, f)}\right)\right]^{2 / n(n+1)} \rightarrow v_{0}(E, \omega)
$$

$v(E, \omega)=v_{0}(E, \omega) \exp \left[-2 \sum \lambda_{k} \alpha_{k}(f)\right] \geqslant v_{0}\left(E, \omega^{\prime}\right) \exp \left[-2 \sum \lambda_{k} \alpha_{k}\left(f^{\prime}\right)\right]$,

$v\left(E, \omega^{\prime}\right)=v_{0}\left(E, \omega^{\prime}\right) \exp \left[-2 \sum \lambda_{k}^{\prime} \alpha_{k}\left(f^{\prime}\right)\right] \geqslant v_{0}(E, \omega) \exp \left[-2 \sum \lambda_{k}^{\prime} \alpha_{k}(f)\right]$ d'où l'on déduit les relations

$$
\begin{gathered}
\sum_{k=1}^{p} \lambda_{k} \alpha_{k}(f)=\frac{1}{2} \log \frac{v_{0}(E, \omega)}{v(E, \omega)}, \\
\sum_{k=1}^{p}\left(\lambda_{k}^{\prime}-\lambda_{k}\right) \alpha_{k}\left(f^{\prime}\right) \leqslant \frac{1}{2} \log \frac{v(E, \omega)}{v\left(E, \omega^{\prime}\right)} \leqslant \sum_{k=1}^{p}\left(\lambda_{k}^{\prime}-\lambda_{k}\right) \alpha_{k}(f),
\end{gathered}
$$

dont la dernière entraîne $(50)$.

Remarque. Dans le cas $p=2$ on a $E=F_{1}+F_{2}, \alpha_{1}(f)=1-\alpha_{2}(f)$ et l'inégalité (50) se réduit, dans l'hypothèse $\lambda_{1}=\lambda_{1}^{\prime}$ à la suivante

$$
\left(\lambda_{2}^{\prime}-\lambda_{2}\right)\left[\alpha_{2}\left(f^{\prime}\right)-\alpha_{2}(f)\right] \leqslant 0 .
$$

II s'ensuit que lorsque $f=\left(\lambda_{1}, \lambda_{2}\right)$ crô̂t sur $F_{2}$ sans varier sur $F_{1}$, la fonctionnelle $\alpha_{2}(f)$ décroît (au sens large) et on a

De (51) on tire

$$
\begin{array}{lll}
\alpha_{2}(f)=1 & \text { si } & \lambda_{2}<\lambda_{1}-\max _{F_{1}} G\left(z, F_{2}\right), \\
\alpha_{2}(f)=0 & \text { si } & \lambda_{2}>\lambda_{1}+\max _{F_{2}} G\left(z, F_{1}\right) .
\end{array}
$$

$$
\left(\lambda_{2}-\lambda_{1}\right) \alpha_{2}(f)=-\lambda_{1}+\frac{1}{2} \log \frac{v_{0}(E, 1, f)}{v(E, 1, f)}
$$

et on a vu que la fonctionnele $v(E, 1, f)=v(E, \omega)$ est continue par rapport à $f$, donc, si $v_{0}(E, 1, f)=v_{0}(E, \omega)$ l'est aussi, la fonctionnelle $a_{2}(f)$ varie continuement avec $f$.

Retournons au cas général où $E=F_{1}+F_{2}+\ldots+F_{p}$. On a vu qu'à toute fonction $f=\left(\lambda_{1}, \lambda_{2}, \ldots, \lambda_{p}\right)$ correspond un système de $p$ fonctionnelles $a_{1}(f), a_{2}(f), \ldots, a_{p}(f)$ remplissant les conditions (49). Supposons que, inversement, à tout système de $p$ nombres $\alpha_{1}, \alpha_{2}, \ldots, \alpha_{p}$ remplis sant les conditions (49), corresponde une fonction $f=\left(\lambda_{1}, \lambda_{2}, \ldots, \lambda_{p}\right)$ telle que $\left.\alpha_{k}=\alpha_{k}(f), k=1,2, \ldots, p^{9}\right)$.

Considérons le système $\alpha_{1}(0), \alpha_{2}(0), \ldots, \alpha_{p}(0)$ et remarquons que, si $\lambda>0$ est suffisamment petit, le système

$$
a_{1}(0)+\lambda, \alpha_{2}(0)-\lambda, \alpha_{3}(0), \ldots, a_{p}(0)
$$

remplit les conditions (49). Soit $g=\left(l_{1}, l_{2}, \ldots, l_{p}\right)$ une fonction en escalier, définie sur $E$ de manière que

$\alpha_{1}(g)=\alpha_{1}(0)+\lambda, \quad \alpha_{2}(g)=a_{2}(0)-\lambda, \quad \alpha_{k}(g)=\alpha_{k}(0), \quad k=3,4, \ldots, p$. Formons la fonction

$$
F(z)=\left[\varphi\left(z, D_{\infty}, 1, g\right) / \varphi\left(z, D_{\infty}, 1,0\right)\right]^{1 / \lambda} \quad \text { où } \quad D_{\infty}=D_{\infty}(E) .
$$

๑) L'existence d'une telle fonction $f$ sera examinée dans un autre travail. 
Elle est holomorphe dans le domaine $D_{\infty}$, le point $z=\infty$ y compris, continue dans $D_{\infty}+E$ et son module sur le continu $F_{t_{t}}$ est égal à $e^{\boldsymbol{l}_{\boldsymbol{k}} / \lambda}$, car

$$
\left|\varphi\left(z, D_{\infty}, 1, g\right)\right|=e^{l_{k}}, \quad\left|\varphi\left(z, D_{\infty}, 1,0\right)\right|=1 \quad \text { si } \quad z \epsilon F_{k} .
$$

D'après ce qui précède, lorsque $z$ parcourt une fois un contour $C_{k i} \subset D_{\infty}$ entourant le continu $F_{k}$ et n'entourant aucun des continus $F_{i}, i \neq k$, l'argument de $\varphi\left(z, D_{\infty}, 1, g\right)$ croit de $.2 \pi \alpha_{k}(g)$ et celui de $\varphi\left(z, D_{\infty}, 1,0\right)$ augmente de $2 \pi \alpha_{k}(0)$. Par suite, lorsque $z$ parcout $C_{k}$, l'argument de $F(z)$

$1^{0}$ augmente de $2 \pi$ si $k=1$

$2^{\circ}$ diminue de $2 \pi$ si $k=2$

$3^{\circ}$ ne change pas si $k=3,4, \ldots, p$.

Il s'ensuit que la fonction $w=F(z)$ est uniforme et univalente dans le domaine $D_{\infty}(E)$ et représente ce domaine sur une couronne circulaire $r<|w|<R$ pourvue de $p-2$ coupures situées sur des circonférences concentriques (une telle couronne est dite domaine canonique de Koebe).

\section{Travaux cités}

[1] J. Górski, Sur certaines fonctions harmoniques jouissant des propriétés extrémales, Ann. Soc. Pol. Math. 23 (1950), p. 259-271.

[2] F. Leja, Sur les suites de polynômes, les ensembles fermés et la fonotion de Green, Ann. Soc. Pol. Math. 12 (1933), p. 57-71.

[3] - Une généralisation de l'écart et du diamètre transfini d'un ensemble, Ann. Soc. Pol. Math. 22 (1949), p. 35-42.

[4] - Sur une famille de fonctions analytiques extrémales, Ann. Soc. Pol. Math. 25 (1952), p. 1-16.

INSTYTUT MATEMATYOZNY POLSKIEJ AKADEMII NAUK

INSTITUT MATHEMATIQUE DE L'ACADÉMITE POLONAISE DES SOIENCES

\section{Remarks on the stability problem for parabolic equations}

by W. MuaK (Kraków)*

The problem of the stability of solutions of parabolic equations has been investigated by Bellman [1], Prodi [4] and Narasimhan [3].

In the first part of this paper our considerations are based on the generalized Westphal-Prodi theorem given in [2]. In the second part we discuss the stability problem for systems of purely non-linear equations of parabolic type. We apply a theorem concerning the evaluation of solutions of parabolic equations given by J. Szarski in [5].

Part I. 1. Suppose $G$ is an open and bounded region lying in the space $E^{m}$ of points $\left(x_{1}, \ldots, x_{m}\right)$. Denote by $B$ the Cartesian product of $G$ and the interval $(0, \infty), B=G \times(0, \infty)$. We denote the boundary of $G$ by $\Gamma . \bar{B}$ denotes the closure of $B$.

Suppose the sequence of functions $u_{1}(x, t), \ldots, u_{n}(x, t)$ is a solution of the parabolic system ${ }^{1}$ )

(1) $\frac{\partial z_{s}}{\partial t}=F_{s}\left(x, t, z_{1}, \ldots, z_{n}, \frac{\partial z_{s}}{\partial x_{i}}, \frac{\partial^{2} \tilde{r}_{s}}{\partial x_{i} \partial x_{k}}\right) \quad(s=1,2, \ldots, n)$.

We say that $u=\left(u_{1}, \ldots, u_{n}\right)$ is a stable solution of $(1)$ if for every $\varepsilon>0$ there exists such $\delta>0$ that for every solution $v=\left(v_{1}, \ldots, v_{n}\right)$ of (1) such that $u_{i}(x, t)=v_{i}(x, t)$ for $(x, t) \epsilon \Gamma \times\langle 0, \infty)(i=1, \ldots, n)$ and $\left|u_{i}(x, 0)-v_{i}(x, 0)\right|<\delta \quad(i=1, \ldots, n)$ we have the inequalities $\left|u_{i}(x, t)-v_{i}(x, t)\right|<\varepsilon,(x, t) \in B(i=1, \ldots, n)$.

Now we investigate systems of the form

$$
\frac{\partial z_{s}}{\partial t}=L_{s}\left[z_{s}\right]+f_{s}\left(x, t, z_{1}, \ldots, z_{n}\right) \quad(s=1,2, \ldots, n),
$$

where $L_{s}$ is the elliptic differential operator of the form

$$
L_{s}[v]=\sum_{i, k=1}^{m} a_{i k}^{s}(x) \frac{\partial^{2} v}{\partial x_{i} \partial x_{k}},
$$

* I wish to express here my thanks to J. Szarski for reading the manuseript of this paper and for his valuable remarks.

1) On the definition of the parabolic system see [2] and [5]. Our systems are normal parabolic systems. 